\title{
Efficient Delivery of Dengue Virus Subunit Vaccines to the Skin by Microprojection Arrays
}

\author{
David A. Muller ${ }^{1,2, *}$, Alexandra C. I. Depelsenaire ${ }^{2}$, Ashleigh E. Shannon ${ }^{1}{ }^{\mathbb{D}}$, Daniel Watterson ${ }^{1}$, \\ Simon R. Corrie ${ }^{3}$, Nick S. Owens ${ }^{2}$, Christiana Agyei-Yeboah ${ }^{1,2}$, Stacey T. M. Cheung ${ }^{1}$, \\ Jin Zhang ${ }^{2}$, Germain J. P. Fernando ${ }^{1,2} \mathbb{D}$, Mark A. F. Kendall ${ }^{2,+}$ and Paul R. Young 1,2,* \\ 1 Australian Infectious Diseases Research Centre, School of Chemistry and Molecular Biosciences, The \\ University of Queensland, Brisbane, QLD 4072, Australia; ashleigh.shannon@uqconnect.edu.au (A.E.S.); \\ d.watterson@uq.edu.au (D.W.); c.agyeiyeboah@uq.edu.au (C.A.-Y.); s.cheung@uq.edu.au (S.T.M.C.); \\ g.fernando@uq.edu.au (G.J.P.F.) \\ 2 Australian Institute for Bioengineering and Nanotechnology, The University of Queensland, Brisbane, \\ QLD 4072, Australia; depelsenaire@gmail.com (A.C.I.D.); nick.s.owens@gmail.com (N.S.O.); \\ cliffjenny@gmail.com (J.Z.); Mark.Kendall@anu.edu.au (M.A.F.K.) \\ 3 Department of Chemical Engineering, ARC Centre of Excellence in Convergent BioNano Science and \\ Technology, Monash University, Clayton, VIC 3800, Australia; simon.corrie@monash.edu \\ * Correspondence: d.muller4@uq.edu.au (D.A.M.); p.young@uq.edu.au (P.R.Y.) \\ † Current Address: Australian National University, Canberra, ACT 2601, Australia.
}

Received: 9 October 2019; Accepted: 14 November 2019; Published: 20 November 2019

\begin{abstract}
Dengue virus is the most important arbovirus impacting global human health, with an estimated 390 million infections annually, and over half the world's population at risk of infection. While significant efforts have been made to develop effective vaccines to mitigate this threat, the task has proven extremely challenging, with new approaches continually being sought. The majority of protective, neutralizing antibodies induced during infection are targeted by the envelope (E) protein, making it an ideal candidate for a subunit vaccine approach. Using truncated, recombinant, secreted E proteins ( $\mathrm{sE}$ ) of all 4 dengue virus serotypes, we have assessed their immunogenicity and protective efficacy in mice, with or without Quil-A as an adjuvant, and delivered via micropatch array (MPA) to the skin in comparison with more traditional routes of immunization. The micropatch contains an ultra-high density array $\left(21,000 / \mathrm{cm}^{2}\right)$ of $110 \mu \mathrm{m}$ microprojections. Mice received 3 doses of $1 \mu \mathrm{g}$ (nanopatch, intradermal, subcutaneous, or intra muscular injection) or $10 \mu \mathrm{g}$ (intradermal, subcutaneous, or intra muscular injection) of tetravalent $\mathrm{sE}$ spaced 4 weeks apart. When adjuvanted with Quil-A, tetravalent $\mathrm{SE}$ vaccination delivered via MPA resulted in earlier induction of virus-neutralizing IgG antibodies for all four serotypes when compared with all of the other vaccination routes. Using the infectious dengue virus AG129 mouse infectious dengue model, these neutralizing antibodies protected all mice from lethal dengue virus type 2 D220 challenge, with protected animals showing no signs of disease or circulating virus. If these results can be translated to humans, MPA-delivered $\mathrm{sE}$ represents a promising approach to dengue virus vaccination.
\end{abstract}

Keywords: dengue virus; nanopatch; secreted E; virus challenge; microneedle; microarray patch; vaccine

\section{Introduction}

Since the re-emergence of dengue virus (DENV) in the 1950's, there has been a steady increase in transmission and global disease over the intervening decades. The increasing geographic range of dengue transmission has resulted in approximately half of the world's population living in dengue 
endemic regions, with an estimated 390 million dengue infections occurring annually [1]. Consequently, dengue viruses are now viewed as the most significant of all disease-causing arbovirus infections.

What makes dengue unique among flaviviruses (including Zika and West Nile virus) is that there are 4 serotypes (serotypes 1-4) that are closely related but antigenically distinct. Patients infected with any of the 4 DENV serotypes can experience a spectrum of clinical outcomes, ranging from asymptomatic to mild febrile illness through to dengue fever. Following primary infection, patients develop a life-long immunity to the original infecting virus serotype [2]. However, upon secondary infection with a heterologous serotype, in a proportion of individuals the induction of cross-reactive antibodies can lead to antibody-dependent enhancement (ADE) of infection and progression to more severe and potentially fatal disease [3].

Given the potential for re-infection with a heterotypic virus serotype leading to enhanced disease outcome, a successful dengue vaccine must be tetravalent, eliciting neutralizing antibodies for all four serotypes, such that naïve individuals are not primed for severe disease from their first encounter with wild-type virus [4]. The first, and so far only licensed DENV vaccine, Dengvaxia (Sanofi Pasteur) [5], is a mix of four chimeric, live-attenuated viruses based on the Yellow Fever virus (YFV) 17D genetic backbone, with the YFV prM and E genes replaced by the equivalent structural genes of each of the 4 DENV serotypes. While early studies proved promising, the vaccine has been shown to have significant limitations, and the WHO now recommends vaccine use be restricted only to recipients who have previously been infected with DENV [6].

Alternative strategies are being actively pursued. The DENV surface glycoprotein E is an ideal candidate for a subunit vaccine, given that it is the major target of a neutralizing antibody response [7-9]. However, ensuring a potent immune response remains a challenge for recombinant subunit vaccines, necessitating an effective adjuvant strategy [10]. Immune responses can be enhanced by targeting antigen presenting cells in the skin by intradermal injection or by use of microarray patches (MAPs), such as the nanopatch.

The nanopatch is a $4 \times 4 \mathrm{~mm}$ ultra-high-density microarray patch (MAP) that contains 21,000 projections $/ \mathrm{cm}^{2}$ of $110 \mu \mathrm{m}$ in length. Vaccine is dry-coated onto the surface of these projections and applied to the skin using a spring-loaded applicator precisely to the epidermal and dermal layers of the skin, containing a high density of antigen presenting cells [11]. As a result of this targeted approach, our group achieved enhanced immune responses with fractional doses as compared to standard needle and syringe injection methods, such as intramuscular and subcutaneous injection. This enhancement was further boosted by the addition of an adjuvant [12]. These enhanced immune responses were observed in live-attenuated [13], DNA [14], inactivated [15,16], virus-like particle [17], conjugated [18], and split-virion vaccines $[19,20]$.

Here, we investigate the utility of several different immunization routes with the nanopatch, namely intramuscular, subcutaneous, intradermal injection, and also intra-cutaneous delivery, for their ability to induce protective immune responses against the dengue secreted $\mathrm{E}(\mathrm{sE})$ protein.

\section{Materials and Methods}

\subsection{Mice}

Female SV129 and AG129 mice (6-8 weeks) were bred in pathogen-free conditions in the Australian Institute for Bioengineering and Nanotechnology (AIBN) animal house. All methods in this study were carried out in accordance with National Health and Medical Research Council guidelines and approved by The University of Queensland Animal Ethics committee (Approval AIBN/556/12 15/10/2014).

\subsection{Cell Lines}

Vero cells were maintained in Optimem media containing 3\% fetal calf serum and penicillin/streptomycin (PenStrep). 


\subsection{DENV1-4 Viral Stocks}

DENV serotypes were propagated in Aedes albopictus C6/36 cells before titration in Vero cells. To titer the virus for plaque reduction neutralization tests (PRNT), DENV viral stocks were serially diluted to 1:10 in optimum serum-free media and incubated for $1 \mathrm{~h}$ at $37^{\circ} \mathrm{C}$ in an atmosphere containing $5 \% \mathrm{CO}_{2}$. Virus was added to confluent monolayers of Vero cells in 96-well plates, seeded the previous day at a density of $4 \times 10^{4}$ cells per well. The virus was allowed to absorb for $1 \mathrm{~h}$ at $37^{\circ} \mathrm{C}$ in $5 \% \mathrm{CO}_{2}$. The virus was removed before addition of 1.5\% carboxymethylcellulose (CMC) overlay with M199 media (Gibco, Grand Island, NY, USA), supplemented with 2\% heat-inactivated fetal bovine serum (FBS). Plates were incubated at $37^{\circ} \mathrm{C}, 5 \% \mathrm{CO}_{2}$ for 2 days. Cells were immunostained as described in the PRNT protocol.

\subsection{Quantification of Mouse Viral Load by DENV Plaque Assay}

Levels of free circulating virus were determined by viral plaque assay performed in Vero cells in 96-well plates. Vero cells were plated at $2 \times 10^{5}$ cells $/ \mathrm{mL}$ at a volume of $200 \mu \mathrm{L}$ and allow to grow to confluency overnight at $37^{\circ} \mathrm{C}$. Cells were washed with phosphate-buffered saline (PBS) followed by serum free media. Plasma samples were serially diluted from $10^{-2}$ to $10^{-8}$ in a separate 96-well plate. After serum free media was removed from cells, $50 \mu \mathrm{L}$ of the serially diluted virus was added to the cells and incubated at $37^{\circ} \mathrm{C}$ for $2 \mathrm{~h}$. Following incubation, media was removed and cells were overlaid with $200 \mu \mathrm{L}$ of $1.5 \%$ carboxymethlycellulose (CMC) in M199 (Invitrogen) with $2.5 \%$ FCS and incubated for 4 days at $37^{\circ} \mathrm{C}$. After 4 days, the overlay was removed and cells were washed with PBS. Cells were then fixed with $200 \mu \mathrm{L}$ of ice-cold $80 \%$ acetone/20\% PBS for $20 \mathrm{~min}$ at $-20{ }^{\circ} \mathrm{C}$. The fixative was removed and plates were dried overnight.

Cells were then washed in PBS/0.1\% bovine serum albumin (BSA) and blocked for 20 min on a shaker at room temperature in the same buffer. Following blocking, $50 \mu \mathrm{L}$ of a 1:200 dilution of an anti-nonstructural protein 1 (NS1) rabbit polyclonal serum diluted in blocking buffer was added to each well and incubated for $1 \mathrm{~h}$ at room temperature. After incubation with the primary antibody, the fixed cells were washed twice in blocking buffer for $3 \mathrm{~min}$ on a shaker. Plates were inverted and tapped to remove wash solution between each wash step. Cells were probed with anti-rabbit Immunoglobulin $\mathrm{G}(\mathrm{IgG})$ secondary antibody (1:2000) conjugated with an 800 fluorophore. Cells were washed in phosphate-buffered saline $0.05 \%$ Tween-20 (PBS-T) 3 times and plates were allowed to dry completely before visualization and imaging on the Odyssey CLx machine (Li-Cor Biotechnology, Lincoln, CA, USA).

\subsection{PRNT Protocol}

PRNT protocol was performed as previously described with some modifications for individual viruses [21]. Vero cells were seeded in 96-well plates at a density of $4 \times 10^{4}$ cells per well and incubated overnight at $37^{\circ} \mathrm{C}$ in $5 \% \mathrm{CO}_{2}$. Mouse sera collected during the study were heat inactivated at $56^{\circ} \mathrm{C}$ for $30 \mathrm{~min}$. Sera were diluted 1:25 in optimum serum-free media and serially diluted to 1:2 in a 96-well plate to a final volume of $60 \mu \mathrm{L}$. An equal volume of DENV virus stock diluted to a final concentration to obtain $\sim 75$ plaque forming unit (PFU)/well was added to each well to give a final serum dilution of 1:50. The serum-virus mixture was incubated at $37{ }^{\circ} \mathrm{C}$ in $5 \% \mathrm{CO}_{2}$ for $1 \mathrm{~h}$ before adding $50 \mu \mathrm{L}$ to confluent Vero cells. Plates were incubated for $1 \mathrm{~h}$ at $37^{\circ} \mathrm{C}$ in $5 \% \mathrm{CO}_{2}$ to allow virus absorption. Virus-serum inoculum was flicked-off and cells were washed twice in PBS before addition of 1.5\% $\mathrm{CMC}$ overlay with M199 and $2 \% \mathrm{FBS}$. Plates were incubated at $37^{\circ} \mathrm{C}, 5 \% \mathrm{CO}_{2}$ for 2 days. This process was repeated for all four serotypes of DENV (DENV1 ET00.243, DENV2 ET00.300, DENV3 ET00.209, and DENV4 ET00.288) in duplicate for each serum sample.

CMC overlay was removed and cells were fixed in ice-cold $80 \%$ acetone/20\% PBS for $15 \mathrm{~min}$ at $-20{ }^{\circ} \mathrm{C}$. Plates were dried completely before blocking for $30 \mathrm{~min}$ at room temperature in milk diluent/blocking solution (KPL) diluted in PBS 0.05\% Tween 20 (PBS-T). Cells were incubated for $1 \mathrm{~h}$ at 
$37^{\circ} \mathrm{C}$ with human anti-envelope monoclonal antibodies (MAbs), produced as previously described [22], and diluted in KPL blocking solution/PBS-T. For DENV serotypes 1, 2, and 3, the MAb 4E11 (1:1000) was used, while DENV4 was incubated with MAb 5H2 (1:500). Cells were washed 3 times in PBS-T and incubated with anti-human IgG secondary antibody (1:2000) conjugated with an 800 fluorophore. Cells were washed in PBS-T 3 times and plates were allowed to dry completely before visualization and imaging were performed on the Odyssey CLx machine.

\subsection{Nanopatch Fabrication and Application}

Silicon nanopatches (NP, $4 \mathrm{~mm}^{2}, 21,000$ projections $/ \mathrm{cm}^{2}$ at $110 \mu \mathrm{m}$ in length) were fabricated at the Melbourne Centre for Nanofabrication, as previously described by Jenkins et al. [23]. Vaccine and adjuvant were formulated with a $1 \%$ methycellulose and $1 \%$ trehalose solution under a nitrogen jet stream, as described by Chen et al. [24]. Coating morphology and removal were characterized by scanning electron microscopy (SEM) (Joel Neoscope, Toyko, Japan), as described by Crichton et al. [11]

\subsection{Vaccine Delivery Efficiency}

To determine the skin-delivered dose by nanopatch, we performed a ${ }^{14} \mathrm{C}$ tracer assay, as described by Fernando et al. (2012) [12].

\subsection{Immunization Study}

The immunization regime was based on similar studies performed investigating candidate dengue vaccines in mouse models. Under ketamine hydrochloride (Ceva Animal Health, Glenorie, Australia) and xylazine hydrochloride (Troy Laboratories, Gendenning, Australia) sedation, SV129 mice were immunized with 1 or $0.1 \mu \mathrm{g}$ tetravalent sE (DENV1: 258848, DENV2: PR159, DENV3: CH53489, DENV4: H241 produced from S2 cells, donated by Prof Mathew Cooper, UQ) formulation by nanopatch or 1-10 $\mu$ g tetravalent sE by intradermal (ID), subcutaneous (SC), or intra muscular (IM) injection, with or without $3 \mu \mathrm{g}$ of the saponin adjuvant Quil-A (Brenntag, Essen, Germany). Control groups received PBS delivered via each investigated injection method, while nanopatch control groups received excipients only. Nanopatches containing the dose to be delivered were applied to each ventral ear pinnae using a proprietary applicator at a velocity of $3.1 \mathrm{~ms}^{-1}$ and kept in place for 2 min. Mice received three doses by nanopatch or SC, ID, or IM injection at 28-day intervals, with blood samples collected one day before vaccination and 28 days after final dose.

\subsection{DENV Challenge Study}

AG129 mice received 3 doses, 4 weeks apart, of $1 \mu \mathrm{g}$ of tetravalent sE delivered by nanopatch or ID injection, as described above. Naïve and virus control groups received PBS via ID injection while nanopatch control groups received excipients only. All groups AG129 mice were challenged 14 days after the final vaccination with $1 \times 10^{4}$ PFU or $1 \times 10^{5}$ PFU of mouse-adapted dengue 2 virus D220 (donated by Prof Eva Harris, UC Berkeley School of Public Health), except the naïve control, which remained uninfected as a control for mouse behavior and wellbeing. Following challenge, tail tip bleeds were collected daily for 10 days and mice were monitored twice daily for weight loss and signs of distress.

\subsection{Anti-sE IgG Enzyme Linked Immunosorbent Assay}

Subunit sE proteins of each of the 4 dengue serotypes were individually coated on to Nunc Maxisorp plates overnight at $4{ }^{\circ} \mathrm{C}$ in $50 \mu \mathrm{L}$ of carbonate-bicarbonate buffer ( $\left.\mathrm{pH} 9.6\right)$ at $100 \mathrm{ng} / \mathrm{mL}$. Plates were then blocked for $1 \mathrm{~h}$ at room temperature with $200 \mu \mathrm{L} 1$ x milk serum diluent (KPL, Inc., Gaithersburg, MD, USA) with $1 \%$ sucrose. Samples serially diluted in blocking buffer containing $0.05 \%$ PBS/Tween $20(50 \mu \mathrm{L})$ were added to blocked plates and incubated for $1 \mathrm{~h}$ at $37^{\circ} \mathrm{C}$, and then washed 6 times in PBS-T. Goat anti-mouse Horseradish Peroxidase (HRP) $(50 \mu \mathrm{L})$ diluted to 1:500 in blocking buffer with PBS-T was added to plates and incubated for $1 \mathrm{~h}$ at $37^{\circ} \mathrm{C}$. Following this incubation, plates 
were washed 6 times in PBS-T then developed using $50 \mu \mathrm{L}$ of 3,3',5,5'-tetramethlybenzidine (TMB) (ELISA systems) for $10 \mathrm{~min}$ and protected from light. The reaction was stopped by the addition of $50 \mu \mathrm{L}$ of $1 \mathrm{M}$ phosphoric acid and absorbance was read at $450 \mathrm{~nm}$.

\subsection{NS1 Quantification}

The quantification of NS1 was performed as originally described by Young et al. [25], with modifications outlined by Muller et al. [26].

\subsection{Statistical Analysis}

All statistical analyses were performed using GraphPad Prism version 6.0f (San Diego, CA, USA). Multiple comparison analysis was performed using one-way analysis of variance (ANOVA), with the alpha level set at 0.05 and with a Tukey post-test.

\section{Results}

\subsection{Nanopatch Coating and Delivery}

Nanopatches were coated with sE protein vaccine formulated in methylcellulose and trehalose to enhance viscosity and protect the vaccine formulation during drying, respectively. To achieve the desired delivery of $1 \mu \mathrm{g}$ of the tetravalent $\mathrm{sE}$ formulation, a ${ }^{14} \mathrm{C}$ tracer was added to the formulation to allow for quantitation of bulk coating deposition into the skin. Figure 1a shows the regular array of $110 \mu \mathrm{m}$ microneedles on the surface of the nanopatch. A representative image of sE coated nanopatches is shown in Figure 1b, with the coated vaccine observed at the tips of the needles. These coated nanopatches were applied to the ear of mice $(n=5)$ to characterize sE delivery. A representative scanning electron microscope (SEM) image taken immediately after application is shown in Figure 1c, confirming the removal of the $\mathrm{sE}$ coating formulation from the tips of the microneedles. Transfer of the ${ }^{14} \mathrm{C}$ tracer to skin was found to be $10 \%$ and $20 \%$ of the total amount loaded onto the patches for formulations, with and without Quil-A, respectively, as seen in Figure $1 d$, for both the formulations delivering a total of $1 \mu \mathrm{g}$ sE $(0.25 \mu \mathrm{g}$ of each DENV serotype sE). Following the development of the coating and delivery conditions, we proceeded to test the immunogenicity of the vaccine in the SV129 mouse model.
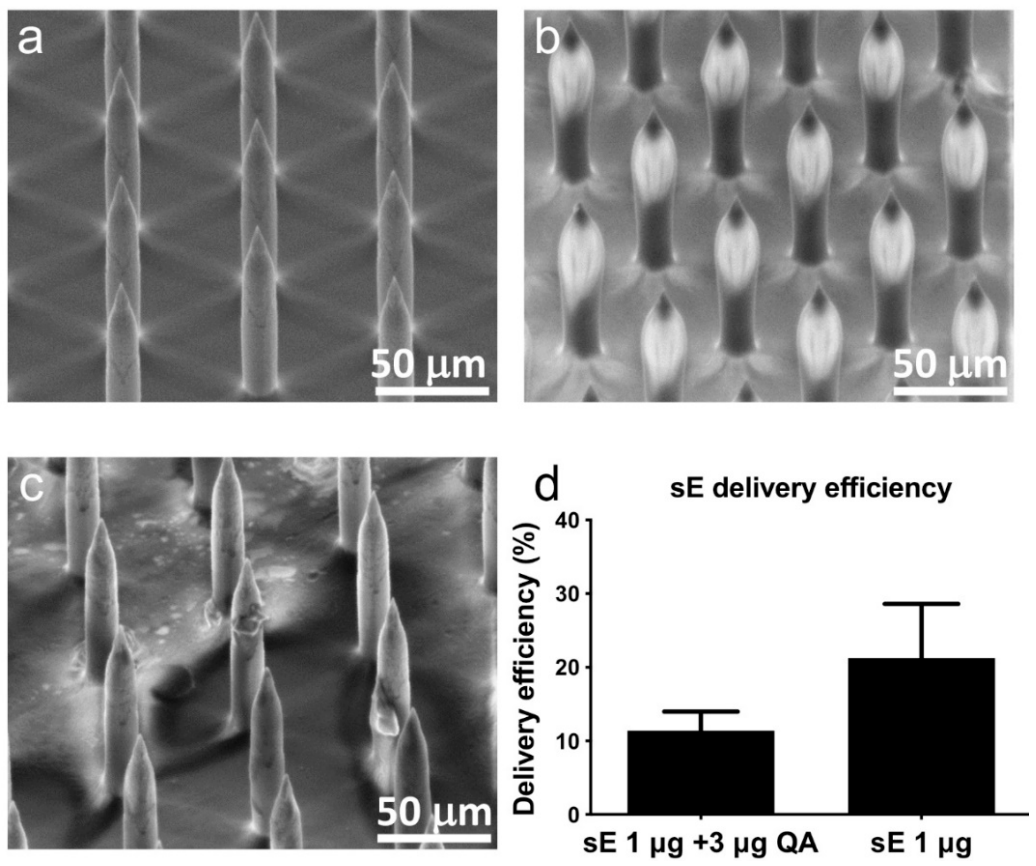

Figure 1. Scanning electron microscopy (SEM) analysis of (a) uncoated microneedles on the surface of the nanopatch: (b) sE coated microneedles; (c) the nanopatch post application, showing the removal of 
vaccine from the tips of the microneedle array. (d) As a measure of delivery efficiency, a ${ }^{14} \mathrm{C}$ tracer was added to the vaccine solution to measure the bulk transfer of the nanopatch vaccine coating to the ear. The bar graph represents mean of 4-5 samples, with error bars indicating standard deviation of the mean. Note: $\mathrm{sE}=$ secreted $\mathrm{E}$ protein.

\section{2. sE Immunization of SV129 Mice}

Immunocompetent SV129 mice (the parental mouse line to AG129 mice) were immunized three times, 21 days apart, with one of two vaccine doses: either $1 \mu \mathrm{g}$ (a mixed dose of $0.25 \mu \mathrm{g}$ each serotype) or $10 \mu \mathrm{g}(2.5 \mu \mathrm{g}$ of each serotype) per $0.05 \mathrm{~mL}$ immunization, with and without Quil-A ( $3 \mu \mathrm{g})$. The 1 and $10 \mu \mathrm{g}$ doses were delivered via intramuscular (IM), intradermal (ID), and subcutaneous (SC) injection with nanopatch delivery of only the $1 \mu \mathrm{g}$ dose. The different immunization strategies were compared for induction of antigen-specific IgG responses (Figure 1 and Figure S1). Overall, similar trends were observed for the immune response to all 4 DENV serotypes (Figure 2a-d). Vaccine co-formulated to deliver $1 \mu \mathrm{g}$ sE and $3 \mu \mathrm{g}$ Quil-A produced significantly higher titers of DENV serotype-specific IgG when delivered by nanopatch or ID injection than by the same dose delivered via IM or SC injection (Figure S1a-d). When the dose was increased to $10 \mu \mathrm{g}$ with $3 \mu \mathrm{g}$ of Quil-A, the mice did not produce significantly greater IgG titers than the $1 \mu \mathrm{g}$ dose. Indeed, significantly lower titers were seen, suggesting the possibility of high doses of sE-induced suppression of the immune response.
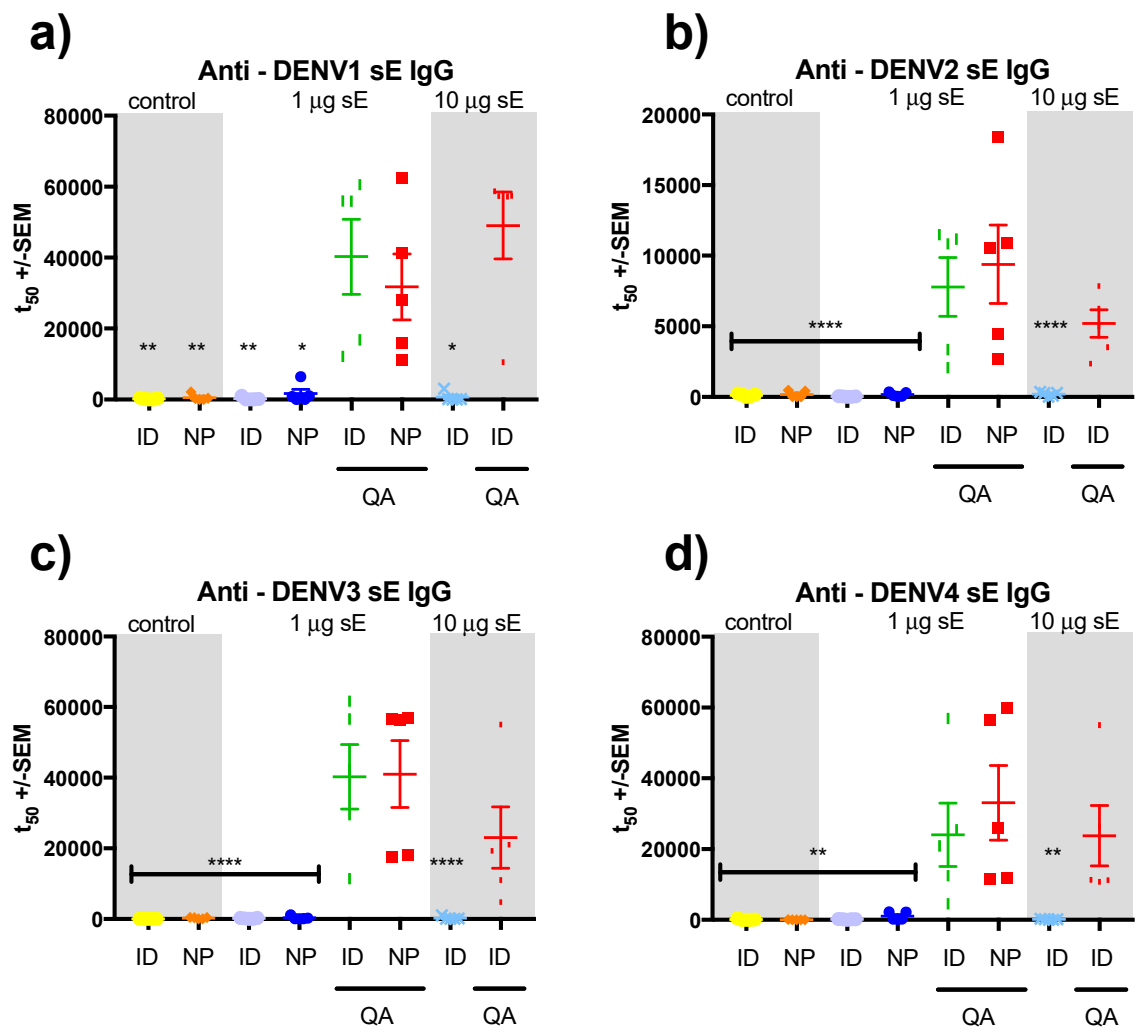

Figure 2. Anti-(sE) Immunoglobulin G (IgG )titers 21 days after the third immunization for SV129 mice vaccinated by nanopatch, and ID injection, with and without the adjuvant Quil-A. Samples were analyzed by anti-sE indirect Enyme-linked immunosorbent assays (ELISA) with midpoint titers shown for each serotype of DENV: (a) dengue 1 anti-sE responses; (b) dengue 2 anti-sE responses; (c) dengue 3 anti-sE IgG responses; and (d) dengue 4 anti-sE IgG responses. Each symbol represents results from a single mouse. Lines indicate mean titers, with bars indicating $+/-$ standard error of the mean. Note: *, **,***, and ${ }^{* * *}$ indicate a statistical significance for the nanopatch Quil-A group, as assessed by one-way analysis of variance (ANOVA, alpha level 0.5), of $p<0.5, p<0.01, p<0.001$ and $p<0.0001$, respectively. 


\section{3. sE Nanopatch Immunization of AG129 Mice}

In order to examine the protective efficacy of the immune responses elicited, we utilized the established AG129 (interferon $\alpha / \beta$, and $\gamma$ receptor knock-outs) mouse challenge model $[27,28]$. The immunogenicity analysis was repeated in AG129 mice $(n=10)$ using all four DENV sE serotypes, and focusing on the comparison between nanopatch and ID delivery routes, given their clear superiority over IM and SC routes (Figure S1), as we have reported previously [29]. We observed (Figure 3a-d) similar levels of antibody responses across all four serotypes, regardless of the delivery route, confirming that the tetravalent antigen induced comparable IgG titers independent of virus serotype. With the change of mouse model, we observed increased antibody titers for those mice immunized without adjuvant, regardless of the delivery route. In the absence of adjuvant, the nanopatch yielded higher antibody titers in comparison to ID, however in the presence of Quil-A, antibody titers were consistent for both nanopatch and ID routes following the final dose (Figures S2-S4).

a)

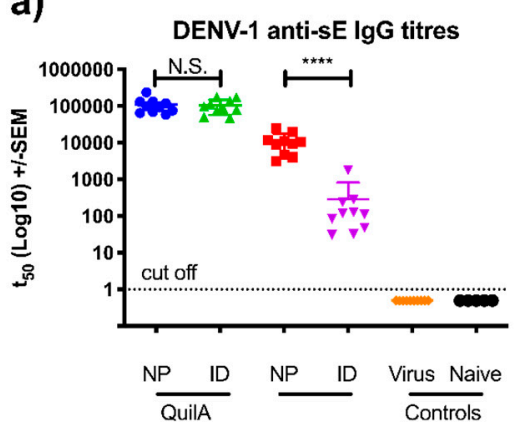

c)

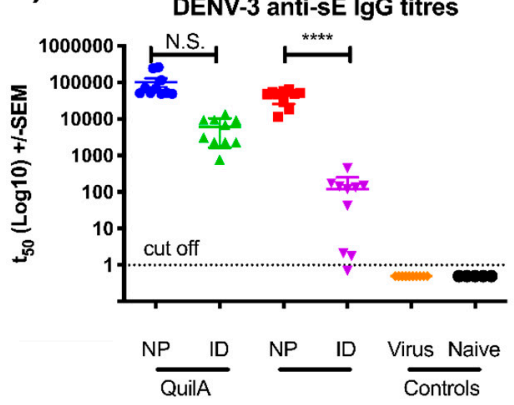

b)

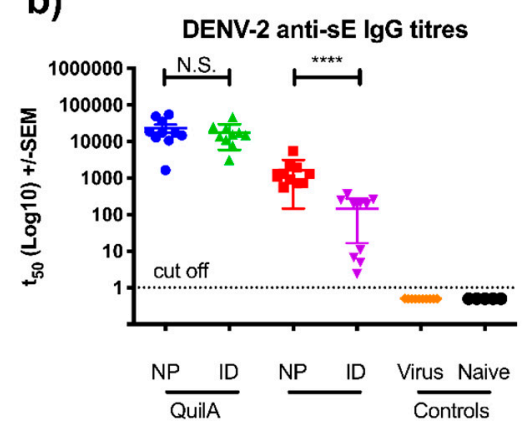

d)

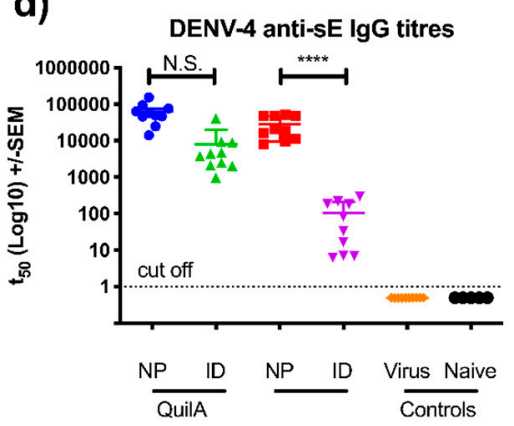

Figure 3. Anti-dengue sE IgG antibody titers for AG129 mice vaccinated by nanopatch and ID injection, with and without the adjuvant Quil-A, determined by indirect ELISA 14 days after the final vaccination. Here, $50 \%$ titers are shown for each serotype of DENV: (a) dengue 1 anti-sE responses; (b) dengue 2 anti-sE responses; (c) dengue 3 anti-sE IgG responses; and (d) dengue 4 anti-sE IgG responses. Each symbol represents a single mouse. Lines indicates mean titers, with bars indicating $+/-$ standard error of the mean. Note: ${ }^{* * *}, * * *$, and ${ }^{* * * *}$ indicate a statistical significance for the nanopatch Quil-A group, as assessed by one-way analysis of variance (ANOVA, alpha level 0.5), of $p<0.05, p<0.01, p<0.001$, and $p<0.0001$, respectively.

In addition to the anti-sE IgG analysis, we performed plaque reduction neutralization (PRNT) assays to determine the presence of virus-neutralizing antibodies. As seen in Figure $4 \mathrm{a}-\mathrm{d}$, the $\mathrm{PRNT}_{80}$ titers showed consistent trends with the anti-sE IgG analysis. Again, vaccination with the tetravalent $\mathrm{sE}$ formulation produced a balanced response, with similar mean neutralizing titers observed for all serotypes. Unlike the IgG analysis, a significant difference was observed between matched ID and nanopatch groups, suggesting a greater proportion of virus-neutralizing antibodies in the antibody response of animals receiving the nanopatch-delivered vaccine. 

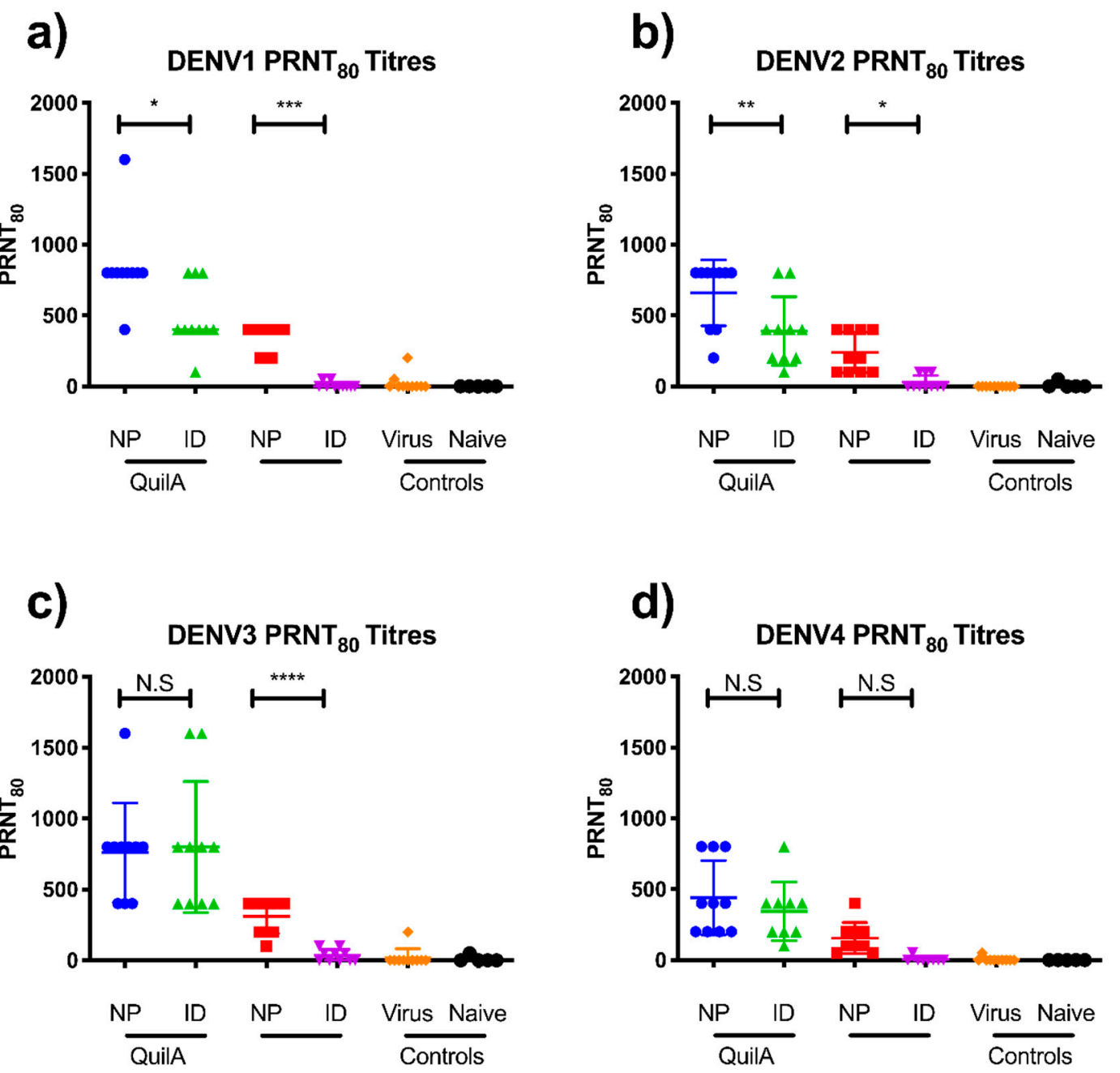

Figure 4. Virus-neutralizing antibody titers for AG129 mice vaccinated by nanopatch and ID injection, with and without the adjuvant Quil-A 14 days after the third vaccination. PRNT $_{80}$ titers are shown for each DENV serotype: (a) DENV1; (b) DENV2; (c) DENV3; and (d) DENV4. Each symbol represents a single mouse. Lines indicate mean titers, with bars indicating $+/-$ standard error of the mean. Note: *, **,***, and ${ }^{* * * *}$ indicate a statistical significance for the nanopatch Quil-A group, as assessed by one-way ANOVA (alpha level 0.5), of $p<0.05, p<0.01, p<0.001$, and $p<0.0001$, respectively.

\subsection{DENV Challenge of Vaccinated AG129 Mice}

Given the strong neutralizing antibody responses induced by nanopatch and ID vaccination in AG129 mice, we next evaluated the degree of protection elicited by a lethal virus challenge. Groups of 10 mice were randomly split into two groups of five for challenge with either $10^{4}$ or $10^{5}$ plaque-forming units (PFU) of the mouse-adapted D220 DENV2 strain (equivalent to one or 10 times the experimentally-determined LD50). Aside from monitoring symptoms of infection by scoring weight loss (Figure S5) and changes in mouse behavior, we measured viral titer and DENV NS1 levels in plasma to determine the effect of vaccination on viral replication. For unvaccinated control mice, challenge with $10^{4}$ or $10^{5}$ PFU resulted in a rapid increase in viral load and NS1 levels over the first 3 days, with mice in a moribund state humanly culled by days $4-5$ (Figure $5 \mathrm{~g}-\mathrm{i}$ ). Mice vaccinated with $\mathrm{sE}$ without adjuvant showed mixed results based on the vaccine delivery route. All mice vaccinated via the ID route succumbed to virus challenge, and had higher circulating viral titers in comparison to the nanopatch-immunized group. In contrast, the nanopatch-immunized group had partial protection from both doses of lethal virus challenge, with significantly lower circulating viral titers (Figure $5 \mathrm{~d}-\mathrm{f}, \mathrm{i}$ ). In the case of adjuvanted vaccinations, $100 \%$ of animals survived 
following nanopatch plus Quil-A immunization, with no virus breakthrough observed (Figure 5i) and significantly reduced NS1 levels (Figure 5j) as compared to the controls (Figure 5a-c). Partial protection was afforded to mice immunized with sE plus adjuvant via the ID route, suggesting that the presence of the adjuvant significantly improved both the immune responses and the degree of protection from lethal virus challenge (Figure 5a). Both viral and NS1 titers were reduced in these groups, consistent with the improved immune response and survival outcome (Figure 5a-c).

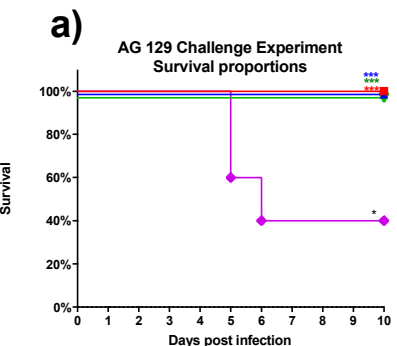

d)

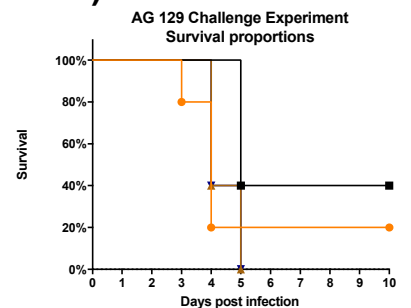

g)

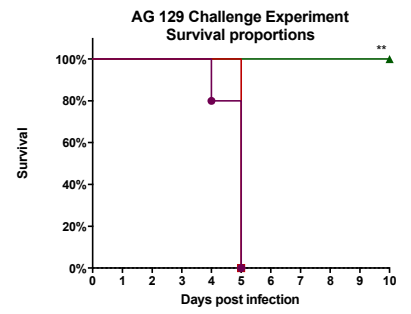

j)

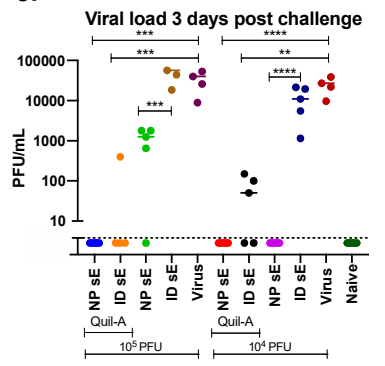

b)

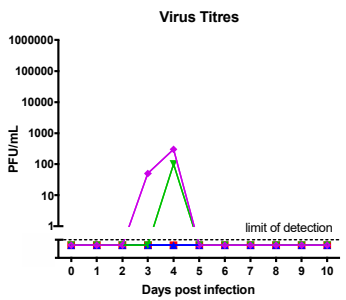

e)

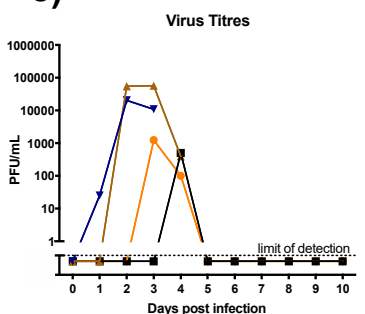

h)

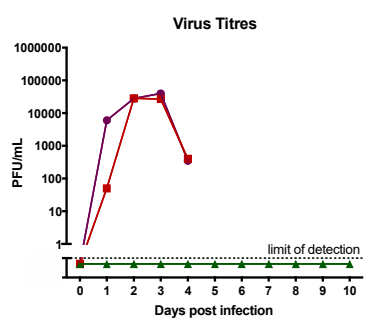

k)

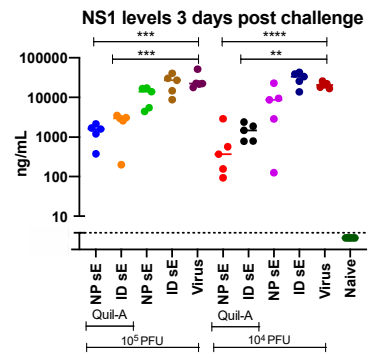

c)

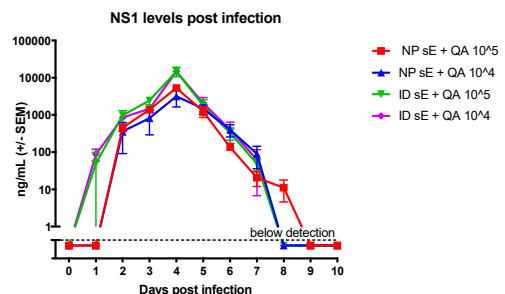

f)

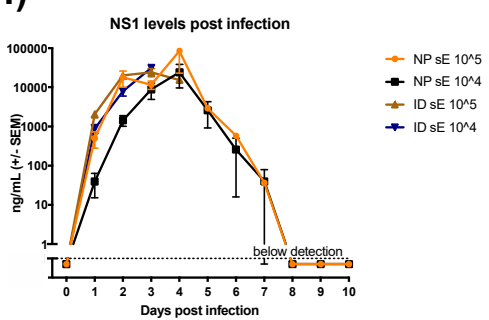

i)

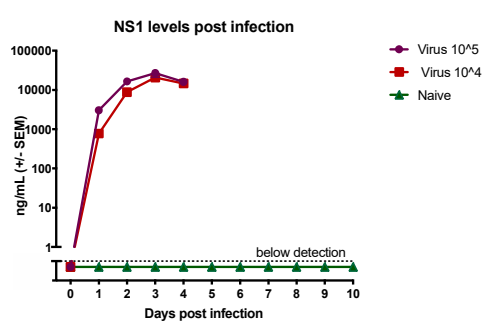

Figure 5. Survival rates, viral titers, and NS1 concentration in mice infected with $1 \times 10^{5}$ or $1 \times 10^{4}$ PFU of DENV2 D220 10 days after the final tetravalent sE vaccination. Mice were monitored daily for survival rate $(\mathbf{a}, \mathbf{d}, \mathbf{g})$, with blood samples collected daily from days 1-10 for analysis. The difference between vaccinated groups and virus control groups was determined by log-rank (Mantel-cox test). Daily samples were analyzed for the presence of circulating virus by plaque assay in Vero cells (b,e,h). Plasma levels of NS1 were also determined by quantitative capture ELISA $(\mathbf{c}, \mathbf{f}, \mathbf{i})$. To demonstrate the significant reduction in virus titer $(\mathbf{j})$ and NS1 titers $(\mathbf{k})$ resulting from vaccination, the raw data for day 3 post-virus challenge is presented. Note: ${ }^{*}, * * * *$ and ${ }^{* * *}$ indicate a statistically significant reduction from the in virus titer and NS1 titers, as assessed by one-way ANOVA (alpha level 0.5). of $p<0.05$, $p<0.01, p<0.001$, and $p<0.0001$, respectively. 


\section{Discussion}

Here, we describe a potent, tetravalent dengue subunit vaccine that induces enhanced levels of neutralizing antibody to all 4 DENV serotypes when delivered by nanopatch compared to other traditional immunization routes. Mice immunized by nanopatch with $1 \mu \mathrm{g}$ of tetravalent $\mathrm{sE}$ formulated with the adjuvant Quil-A were afforded complete protection in a mouse challenge model. If this approach in mice translates to similar efficacy in humans, this could result in a highly protective vaccine coupled with the practical advantages conferred by the nanopatch delivery platform.

The licensing of the Sanofi dengue chimeric vaccine was an important step in dengue vaccine development. However, given the age restrictions and specific conditions under which the vaccine has been recommended for use, there remains an urgent need for further DENV vaccine development. An array of approaches are currently underway, with additional chimeric vaccines in advanced development by Takeda and National Institutes of Health. Subunit vaccine approaches based on the main target of a neutralizing antibody response, the DENV E glycoprotein, also have great potential [7]. Despite previous evidence that the DENV E protein is poorly immunogenic on its own $[7,10]$, there are several practical advantages in the subunit vaccine approach over live-attenuated vaccines. These include safety, flexibility to adjust the antigenic dose to balance the immune response, and the ability to safely immunize infants and immunosuppressed individuals [30].

To overcome the weakly immunogenic nature of the E protein, we included Quil-A adjuvant, which we have previously shown significantly enhances immune responses in small animal models $[12,18,31,32]$. The formulated vaccines were then dry-coated on the nanopatch for delivery. The nanopatch provides several advantages over traditional needle and syringe delivery, particularly in developing world and resource-limited settings. These include the absence of a need for specialized medical personnel to administer the vaccine, no sharp waste being generated, and no need for vaccine reconstitution [33].

In SV129 mice, a balanced IgG response was observed for DENV1, DENV3, and DENV4, however IgG titers were significantly lower in mice immunized with DENV2 sE. This finding is in contrast with a previous study carried out in BALB/c mice using intramuscular immunization of sE, which reported a more balanced IgG response with a vaccine formulation comprising a molar ratio of 1:1:1:2 of DENV1/2/3/4 sE, respectively [34,35]. The serotype bias differences between these studies may be due to mouse strain variation, given that the switch to the AG129 mouse model led to a more balanced IgG immune response following immunization with an equimolar amount of DENV1-4 sE. Immunization with $1 \mu \mathrm{g}$ tetravalent $\mathrm{sE}$ with and without Quil-A elicited similar antibody titers against all dengue serotypes using either NP or ID routes. As seen previously, Quil-A significantly increased both IgG and neutralization titers (Figures 3 and 4). Quil-A is a highly effective adjuvant in small animal models and is used here to assess the role of adjuvants in eliciting an effective protective immune response. However, Quil-A is not suitable for vaccine use in humans, and alternative adjuvants will be screened in future studies. These include QS-21, a purified derivative of Quil-A that is licensed for use in conjunction with monophosphoryl lipid A (AS01b) as an adjuvant to the shingles vaccine, Shingerix [36]. To translate our findings beyond these preliminary preclinical evaluation studies, careful consideration of adjuvant choice will be required. While beyond the scope of this study, DENV serotype antigen ratios and epitope bias will need to be evaluated to ensure a balanced, elicited antibody response that takes into consideration the important issue of ADE.

While there has been intensive research over many decades characterizing the anti-dengue immune response to various candidate dengue vaccines, there have only been a limited number of studies performed using interferon receptor knockout AG129 (interferon $\alpha / \beta, \gamma$ receptor knockout) or interferon $\alpha / \beta$ receptor knockout (INFAR1) mice. The attraction of these interferon receptor knockout mice are that they are receptive to DENV infection via peripheral challenge with resulting viremia and relevant pathology. However, their IFN-deficient status presents some caveats to the interpretation of immune responses. Nevertheless, immune responses elicited in these animal models to various vaccine formulations have previously been shown to induce some level of protection from DENV 
challenge [37,38]. To the best of our knowledge, the current study is the first to assess the potential of sE subunit formulations to afford protection from DENV challenge in the AG129 mouse model. Mice immunized by NP delivery with sE and formulated with Quil-A showed no signs of virus infection, with all mice surviving. Furthermore, upon analysis of plasma samples collected over the course of infection, no viremia breakthrough was observed. It is notable that NS1 was still detected as circulating in the blood of these protected animals, albeit at slightly reduced levels, and with peak levels delayed by a day when compared to control infected mice. This suggests ongoing virus replication and perhaps further immune stimulation in the absence of detectable virus circulation.

This is the first study to demonstrate effective microarray patch delivery of a tetravalent DENV $\mathrm{sE}$ vaccine. Immune responses to all DENV serotypes were elicited and protection against DENV-2 challenge was demonstrated in the AG129 mouse model. The highly effective nature of the nanopatch array delivery system warrants further evaluation in larger animal models and with consideration for other flaviviruses, including yellow fever and emerging threats such as Zika virus.

Supplementary Materials: The following are available online at http://www.mdpi.com/2076-393X/7/4/189/s1: Figure S1: Complete data set of anti-sE IgG titers from SV129 mice vaccinated by nanopatch, ID, SC, and IM injection, with and without the adjuvant Quli A. Figure S2: AG129 anti-sE IgG ELISA results post dose 1. Figure S3: AG129 anti-sE IgG ELISA results post dose 2. Figure S4: AG129 anti-sE IgG ELISA results post dose 3. Figure S4: Percentage weight loss from AG129 mice following DENV D220 challenge.

Author Contributions: Conceptualization, D.A.M. and P.R.Y.; methodology, D.A.M., A.C.I.D., and A.E.S.; formal analysis, D.A.M., A.C.I.D., and G.J.P.F.; investigation, D.A.M., A.C.I.D., A.E.S., D.W., S.R.C., N.S.O., C.A.-Y., S.T.M.C., and J.Z.; writing-original draft preparation, D.A.M. and P.R.Y.; writing-review and editing, D.A.M., P.R.Y., M.A.F.K., D.W., A.C.I.D., and G.J.P.F.

Funding: All work was funded by an Accelerate Early Career Fellowship from The Queensland State Government partnered with Vaxxas and Merck Research laboratories.

Acknowledgments: We would like to thank Vaxxas and Merck Research laboratories and Queensland State Government for their financial support of this project. Furthermore, we would like to thank Prof Eva Harris for providing the D220 dengue virus type 2. We thank Julian Hickling (Working in Tandem Ltd., UK) for his critical reading of this manuscript. We also acknowledge the technical assistance of Chelsea Stewart and staff of the University of Queensland Biological Resources. This work was performed in part at the Queensland Node of the Australian National Fabrication Facility.

Conflicts of Interest: D.A.M., G.J.P.F., and P.R.Y. are consultants for nanopatch development company Vaxxas. M.A.F.K. declares competing financial interests as the founder of Vaxxas and is the inventor of several patents currently licensed by Vaxxas. The remaining authors declare no conflict. The sponsors had no role in the design, execution, interpretation, or writing of the study.

\section{References}

1. Bhatt, S.; Gething, P.W.; Brady, O.J.; Messina, J.P.; Farlow, A.W.; Moyes, C.L.; Drake, J.M.; Brownstein, J.S.; Hoen, A.G.; Sankoh, O.; et al. The global distribution and burden of dengue. Nature 2013, 496, 504-507. [CrossRef]

2. Halstead, S.B. Etiologies of the experimental dengues of Siler and Simmons. Am. J. Trop. Med. Hyg. 1974, 23, 974-982. [CrossRef]

3. Guzman, M.G.; Gubler, D.J.; Izquierdo, A.; Martinez, E.; Halstead, S.B. Dengue infection. Nat. Rev. Dis. Primers 2016, 2, 16055. [CrossRef] [PubMed]

4. Lim, S.K.; Lee, Y.S.; Namkung, S.; Lim, J.K.; Yoon, I.K. Prospects for dengue vaccines for travelers. Clin. Exp. Vaccine Res. 2016, 5, 89-100. [CrossRef]

5. Ferguson, N.M.; Rodriguez-Barraquer, I.; Dorigatti, I.; Mier, Y.T.-R.L.; Laydon, D.J.; Cummings, D.A. Benefits and risks of the Sanofi-Pasteur dengue vaccine: Modeling optimal deployment. Science 2016, 353, 1033-1036. [CrossRef]

6. WHO. WHO Advises Dengvaxia be used only in People Infected with Dengue. Available online: http://www. who.int/medicines/news/2017/WHO-advises-dengvaxia-used-only-in-people-previously-infected/en/ (accessed on 19 January 2018). 
7. Clements, D.E.; Coller, B.A.; Lieberman, M.M.; Ogata, S.; Wang, G.; Harada, K.E.; Putnak, J.R.; Ivy, J.M.; McDonell, M.; Bignami, G.S.; et al. Development of a recombinant tetravalent dengue virus vaccine: Immunogenicity and efficacy studies in mice and monkeys. Vaccine 2010, 28, 2705-2715. [CrossRef]

8. da Costa, V.G.; Marques-Silva, A.C.; Floriano, V.G.; Moreli, M.L. Safety, immunogenicity and efficacy of a recombinant tetravalent dengue vaccine: A meta-analysis of randomized trials. Vaccine 2014, 32, 4885-4892. [CrossRef]

9. Gouvea, I.E.; Izidoro, M.A.; Judice, W.A.; Cezari, M.H.; Caliendo, G.; Santagada, V.; dos Santos, C.N.; Queiroz, M.H.; Juliano, M.A.; Young, P.R.; et al. Substrate specificity of recombinant dengue 2 virus NS2B-NS3 protease: Influence of natural and unnatural basic amino acids on hydrolysis of synthetic fluorescent substrates. Arch. Biochem. Biophys. 2007, 457, 187-196. [CrossRef]

10. Swaminathan, G.; Thoryk, E.A.; Cox, K.S.; Smith, J.S.; Wolf, J.J.; Gindy, M.E.; Casimiro, D.R.; Bett, A.J. A tetravalent sub-unit dengue vaccine formulated with ionizable cationic lipid nanoparticle induces significant immune responses in rodents and non-human primates. Sci. Rep. 2016, 6, 34215. [CrossRef]

11. Crichton, M.L.; Ansaldo, A.; Chen, X.; Prow, T.W.; Fernando, G.J.; Kendall, M.A. The effect of strain rate on the precision of penetration of short densely-packed microprojection array patches coated with vaccine. Biomaterials 2010, 31, 4562-4572. [CrossRef]

12. Fernando, G.J.; Chen, X.; Primiero, C.A.; Yukiko, S.R.; Fairmaid, E.J.; Corbett, H.J.; Frazer, I.H.; Brown, L.E.; Kendall, M.A. Nanopatch targeted delivery of both antigen and adjuvant to skin synergistically drives enhanced antibody responses. J. Control. Release 2012, 159, 215-221. [CrossRef]

13. Pearson, F.E.; McNeilly, C.L.; Crichton, M.L.; Primiero, C.A.; Yukiko, S.R.; Fernando, G.J.; Chen, X.; Gilbert, S.C.; Hill, A.V.; Kendall, M.A. Dry-coated live viral vector vaccines delivered by nanopatch microprojections retain long-term thermostability and induce transgene-specific $\mathrm{T}$ cell responses in mice. PLoS ONE 2013, 8, e67888. [CrossRef]

14. Prow, T.W.; Chen, X.; Prow, N.A.; Fernando, G.J.; Tan, C.S.; Raphael, A.P.; Chang, D.; Ruutu, M.P.; Jenkins, D.W.; Pyke, A.; et al. Nanopatch-targeted skin vaccination against West Nile Virus and Chikungunya virus in mice. Small 2010, 6, 1776-1784. [CrossRef]

15. Muller, D.A.; Pearson, F.E.; Fernando, G.J.; Agyei-Yeboah, C.; Owens, N.S.; Corrie, S.R.; Crichton, M.L.; Wei, J.C.; Weldon, W.C.; Oberste, M.S.; et al. Inactivated poliovirus type 2 vaccine delivered to rat skin via high density microprojection array elicits potent neutralising antibody responses. Sci. Rep. 2016, 6, 22094. [CrossRef]

16. Muller, D.A.; Fernando, G.J.P.; Owens, N.S.; Agyei-Yeboah, C.; Wei, J.C.J.; Depelsenaire, A.C.I.; Forster, A.; Fahey, P.; Weldon, W.C.; Oberste, M.S.; et al. High-density microprojection array delivery to rat skin of low doses of trivalent inactivated poliovirus vaccine elicits potent neutralising antibody responses. Sci. Rep. 2017, 7, 12644. [CrossRef]

17. Corbett, H.J.; Fernando, G.J.; Chen, X.; Frazer, I.H.; Kendall, M.A. Skin vaccination against cervical cancer associated human papillomavirus with a novel micro-projection array in a mouse model. PLOS ONE 2010, 5, e13460. [CrossRef]

18. Pearson, F.E.; Muller, D.A.; Roalfe, L.; Zancolli, M.; Goldblatt, D.; Kendall, M.A. Functional anti-polysaccharide IgG titres induced by unadjuvanted pneumococcal-conjugate vaccine when delivered by microprojection-based skin patch. Vaccine 2015, 33, 6675-6683. [CrossRef]

19. Crichton, M.L.; Muller, D.A.; Depelsenaire, A.C.; Pearson, F.E.; Wei, J.; Coffey, J.; Zhang, J.; Fernando, G.J.; Kendall, M.A. The changing shape of vaccination: Improving immune responses through geometrical variations of a microdevice for immunization. Sci. Rep. 2016, 6, 27217. [CrossRef]

20. Fernando, G.J.; Chen, X.; Prow, T.W.; Crichton, M.L.; Fairmaid, E.J.; Roberts, M.S.; Frazer, I.H.; Brown, L.E.; Kendall, M.A. Potent immunity to low doses of influenza vaccine by probabilistic guided micro-targeted skin delivery in a mouse model. PLoS ONE 2010, 5, e10266. [CrossRef]

21. Watterson, D.; Robinson, J.; Chappell, K.J.; Butler, M.S.; Edwards, D.J.; Fry, S.R.; Bermingham, I.M.; Cooper, M.A.; Young, P.R. A generic screening platform for inhibitors of virus induced cell fusion using cellular electrical impedance. Sci. Rep. 2016, 6, 22791. [CrossRef]

22. Li, J.; Watterson, D.; Chang, C.W.; Che, X.Y.; Li, X.Q.; Ericsson, D.J.; Qiu, L.W.; Cai, J.P.; Chen, J.; Fry, S.R.; et al. Structural and functional characterization of a cross-reactive dengue virus neutralizing antibody that recognizes a cryptic epitope. Structure 2018, 26, 51e54-59e54. [CrossRef] 
23. Jenkins, D.; Corrie, S.R.; Flaim, C.; Kendall, M.A. High density and high aspect ratio solid micro-nanoprojection arrays for targeted skin vaccine delivery and specific antibody extraction. RSC Adv. 2012, 2, 3490-3495. [CrossRef]

24. Chen, X.; Kask, A.S.; Crichton, M.L.; McNeilly, C.; Yukiko, S.; Dong, L.; Marshak, J.O.; Jarrahian, C.; Fernando, G.J.; Chen, D.; et al. Improved DNA vaccination by skin-targeted delivery using dry-coated densely-packed microprojection arrays. J. Control. Release 2010, 148, 327-333. [CrossRef]

25. Young, P.R. Antigenic analysis of dengue virus using monoclonal antibodies. Southeast Asian J. Trop. Med. Public Health 1990, 21, 646-651.

26. Muller, D.A.; Corrie, S.R.; Coffey, J.; Young, P.R.; Kendall, M.A. Surface modified microprojection arrays for the selective extraction of the dengue virus NS1 protein as a marker for disease. Anal. Chem. 2012, 84, 3262-3268. [CrossRef]

27. Sarathy, V.V.; Milligan, G.N.; Bourne, N.; Barrett, A.D. Mouse models of dengue virus infection for vaccine testing. Vaccine 2015, 33, 7051-7060. [CrossRef]

28. Zellweger, R.M.; Shresta, S. Mouse models to study dengue virus immunology and pathogenesis. Front. Immunol. 2014, 5, 151. [CrossRef]

29. Depelsenaire, A.C.; Meliga, S.C.; McNeilly, C.L.; Pearson, F.E.; Coffey, J.W.; Haigh, O.L.; Flaim, C.J.; Frazer, I.H.; Kendall, M.A. Colocalization of cell death with antigen deposition in skin enhances vaccine immunogenicity. J. Investig. Dermatol. 2014, 134, 2361-2370. [CrossRef]

30. Moyle, P.M. Biotechnology approaches to produce potent, self-adjuvanting antigen-adjuvant fusion protein subunit vaccines. Biotechnol. Adv. 2017, 35, 375-389. [CrossRef]

31. Ng, H.I.; Fernando, G.J.; Depelsenaire, A.C.; Kendall, M.A. Potent response of QS-21 as a vaccine adjuvant in the skin when delivered with the Nanopatch, resulted in adjuvant dose sparing. Sci. Rep. 2016, 6, 29368. [CrossRef]

32. Ng, H.I.; Tuong, Z.K.; Fernando, G.J.P.; Depelsenaire, A.C.I.; Meliga, S.C.; Frazer, I.H.; Kendall, M.A.F. Microprojection arrays applied to skin generate mechanical stress, induce an inflammatory transcriptome and cell death, and improve vaccine-induced immune responses. NPJ Vaccines 2019, 4, 41. [CrossRef] [PubMed]

33. Chen, X.; Fernando, G.J.; Crichton, M.L.; Flaim, C.; Yukiko, S.R.; Fairmaid, E.J.; Corbett, H.J.; Primiero, C.A.; Ansaldo, A.B.; Frazer, I.H.; et al. Improving the reach of vaccines to low-resource regions, with a needle-free vaccine delivery device and long-term thermostabilization. J. Control. Release 2011, 152, 349-355. [CrossRef]

34. Govindarajan, D.; Meschino, S.; Guan, L.M.; Clements, D.E.; ter Meulenc, J.H.; Casimiro, D.R.; Collera, B.A.G.; Bett, A.J. Preclinical development of a dengue tetravalent recombinant subunit vaccine: Immunogenicity and protective efficacy in nonhuman primates. Vaccine 2015, 33, 4105-4116. [CrossRef] [PubMed]

35. Coller, B.A.; Clements, D.E.; Bett, A.J.; Sagar, S.L.; Ter Meulen, J.H. The development of recombinant subunit envelope-based vaccines to protect against dengue virus induced disease. Vaccine 2011, 29, 7267-7275. [CrossRef] [PubMed]

36. Didierlaurent, A.M.; Laupeze, B.; Di Pasquale, A.; Hergli, N.; Collignon, C.; Garcon, N. Adjuvant system AS01: Helping to overcome the challenges of modern vaccines. Expert Rev. Vaccines 2017, 16, 55-63. [CrossRef]

37. Brewoo, J.N.; Kinney, R.M.; Powell, T.D.; Arguello, J.J.; Silengo, S.J.; Partidos, C.D.; Huang, C.Y.; Stinchcomb, D.T.; Osorio, J.E. Immunogenicity and efficacy of chimeric dengue vaccine (DENVax) formulations in interferon-deficient AG129 mice. Vaccine 2012, 30, 1513-1520. [CrossRef]

38. Hertz, T.; Beatty, P.R.; MacMillen, Z.; Killingbeck, S.S.; Wang, C.; Harris, E. Antibody epitopes identified in critical regions of dengue virus nonstructural 1 protein in mouse vaccination and natural human infections. J. Immunol. 2017, 198, 4025-4035. [CrossRef]

(C) 2019 by the authors. Licensee MDPI, Basel, Switzerland. This article is an open access article distributed under the terms and conditions of the Creative Commons Attribution (CC BY) license (http://creativecommons.org/licenses/by/4.0/). 